\title{
DETERMINACIÓN DE LOS HONORARIOS DEL ÁRBITRO DESDE LA ÓPTICA OBLIGACIONAL: PROBLEMATIZACIÓN DEL ESTADIO ACTUAL Y PROPUESTA DE SOLUCIONES.
}

\author{
María Fernanda Vásquez Palma*
}

\section{De la relación habida entre las partes y el árbitro en un juicio arbitral.}

Esta relación se forja a partir de un pacto realizado entre las partes que designan al árbitro, ya sea de manera directa o indirecta (Ej. por nombramiento judicial), y el árbitro que acepta el encargo, de ella surgen derechos y obligaciones para ambos. El legislador chileno no señala cuál es la naturaleza de este contrato, si es una estricta relación profesional de la labor desempeñada por el árbitro, si se trata de un contrato de mandato u otro; por esta razón ha sido la doctrina chilena la que ha denominado a esta relación comúnmente como "contrato de compromisario", entendiendo por tal aquél contrato por el cual una persona se obliga a desempeñar las funciones de árbitro ante otras que litigan ante él y que se obligan a remunerarle sus servicios con unos honorarios ${ }^{1}$. Junto a ella se encuentra la tesis del contrato del mandato ${ }^{2}$,

Profesora de Derecho Comercial, Facultad de Ciencias Jurídicas y Sociales de la Universidad de Talca. Doctora en Derecho por la Universidad Complutense de Madrid. Magíster en Derecho Privado por la Universidad de Talca. Correo electrónico: mfvasquez@utalca.cl

$1 \quad C f$. P. Aylwin Azocar, El juicio arbitral, Ed. Jurídica de Chile, 2005, p. 352; R. Abeliuk Manasevic, "El arbitraje y las obligaciones de las partes y del árbitro", Estudios de arbitraje, Libro Homenaje a Patricio Aylwin Azocar, Ed. Jurídica de Chile, 2007, pp. pp.41-53.

2 La jurisprudencia, sin ser radical, ni concluyente, ha optado por la tesis del mandato, así por ejemplo, la CS con fecha 23 de septiembre de 1993, en causa "Corporación Frutícola Chilena Ltda." señala sobre el particular que: "Si a la fecha de la petición de aclaración del fallo dictado por el Árbitro, el mandato de éste había expirado, tanto por haber, vencido el plazo legal que tenía para evacuar su encargo, cuanto por haber cumplido efectivamente con el mandato que le fuera conferido por los interesados, al dictar la sentencia definitiva en la fecha ya señalada, el árbitro no tenía jurisdicción para conocer de la expresada solicitud de aclaración, que le fuere formulada por una de las partes del pleito, porque ya no existía órgano jurisdiccional alguno para resolver el expresado recurso de aclaración, por haberse extinguido el término legal para el desempeño del mandato hecho al Árbitro, y por el cumplimiento efectivo del encargo por parte de éste." 
cuya misión del mandatario se centra en decidir las cuestiones pendientes entre las partes, quedando obligado a cumplir el encargo con prohibición de traspasar sus límites, y el deber de las partes de indemnizarle por los daños y perjuicios que le hubiere causado el incumplimiento del mandato. Igualmente se plantea la tesis del contrato de prestación de servicios, que puede ser oneroso o gratuito dependiendo de lo convenido en relación a los honorarios, e inclusive se ha llegado a manifestar que estos honorarios corresponden a las costas personales.

Con anterioridad nos hemos ocupado de este tema restando valor a dichas elucubraciones, en el convencimiento que el árbitro no es un mandatario tradicional al servicio del interés de los litigantes, ni es un prestador de servicios que actúe por cuenta o encargo de las partes, ni menos aún sus honorarios responden al criterio de costas. En efecto, el árbitro pondera un interés que afecta a las partes pero que decide en función de alcanzar un bien de interés superior y público cual es la solución justa del caso que se enjuicia; de esta forma, la relación existente entre el árbitro y las partes no puede responder a un encargo personal, pues ello implicaría la posibilidad de revocarlo sin justa causa, y un nivel de dependencia del árbitro hacia las partes que no se puede dar. Por su parte, es impensable catalogar a los honorarios como simples costas personales pues no se pueden confundir ambas nociones, en tanto los primeros nacen de una relación obligacional entre el árbitro y las partes, mientras que las costas son fijadas a causa de un proceso y su cobro da lugar a un incidente. Se entiende que las costas personales se estiman respecto de los abogados de cada una de las partes del litigio y demás personas que hayan intervenido o prestado servicios en el proceso, no así en relación a las funciones desempeñadas por el árbitro; entenderlo de otra forma, equivaldría a sostener que el árbitro es juez y parte de su propia remuneración, lo que ciertamente no resiste mayor análisis. De esta manera, consideramos que si lo que se otorga es autoridad para resolver conflictos, no podemos limitar a esta relación a un plano pura y típicamente contractual. Tal relación debe explicarse desde una combinación de diversos estadios jurídicos, siendo su motor una relación obligacional atípica en sus inicios ${ }^{3}$.

De la mano de esta falta de tratamiento normativo se encuentran los honorarios del árbitro, pues en este punto nada se señala. A pesar de ello, existe consenso a la hora de considerar que la obligación de las partes de pagar los honorarios de los árbitros que acepta el cargo y tramita un arbitraje, se entiende pertenecer naturalmente al instituto arbitral ${ }^{4}$. El problema de su cuantificación y pago se ocasiona cuando el arbitraje es $a d h o c^{5}$, y las partes de la relación no

\footnotetext{
Para mayor información, Vid., Mª F. Vásquez Palma, Arbitraje en Chile. Análisis crítico de su normativa y jurisprudencia, Ed. Legal Publishing, Santiago, 2009, pp. 173 y sgtes.

Entre otros, Vid. P. Aylwin Azócar, El juicio arbitral, Ed. Jurídica de Chile, $5^{\circ}$ edición, p. 364.

Si el arbitraje es institucional, normalmente los honorarios de los árbitros y los gastos de administración, serán determinados anticipadamente. Sobre las diferenciaciones entre ambas tipologías de arbitraje y sus repercusiones, Vid. Ma. F. Vásquez Palma, Ibid., pp. 68 y sgtes.
} 
Determinación de los honorarios del arbitro desde la óptica obligacional:

Problematización del estadio actual y propuesta de soluciones.

han determinado nada al momento de formalizar la relación con el árbitro, pues en este caso, los honorarios deberán fijarse una vez que el juicio ha finalizado, pues a esa fecha las partes difícilmente querrán hacerse cargo de los honorarios del árbitro, máxime si ha perdido el juicio.

En este caso surgen diversas interrogantes: ¿Cómo y quién fijará los honorarios?, ¿en base a qué criterios?, ¿a quién le corresponde pagar los honorarios, en qué oportunidad y cómo? Dado el silencio legislativo, ha sido la jurisprudencia la que ha debido llenar de contenido de esta obligación, tomando para ello en consideración un par de normas que, desde un punto de vista adjetivo, se pronuncian sobre esta materia. En este camino revisaremos uno de los últimos fallos que se han dictado sobre esta materia ${ }^{6}$, con el objeto que su estudio nos sirva para ilustrar de manera concreta los aludidos problemas y orientar posibles soluciones a las mismas.

\section{Revisión y análisis del fallo: Causa "Niño Morales, Dictinio c/Almonte Puentes, Daniel y otros", $\operatorname{Rol} N^{o} 4.739-07^{7}$.}

Esta sentencia fue dictada por la Corte Suprema con fecha 1 de octubre de 2008, y en lo concreto expuso:

"Corresponde acoger la demanda de honorarios seguida por el árbitro que dictó el laudo arbitral, toda vez que habiéndose cumplido con lo encargado en los autos arbitrales sin haberse fijado la cuantía de su remuneración cabe su valuación por el sentenciador".

En primera instancia el juez competente de Chillán, al resolver un juicio sumario sobre cobro de honorarios, acogió la demanda de pagar $\$ 50.000 .000$ por concepto de honorarios, reajustados según el IPC entre la fecha de la sentencia y la de su pago efectivo, con costas. En contra de dicho fallo, la parte demandada interpuso recurso de apelación y la corte de Apelaciones de Chillán, por sentencia pronunciada el 03 de agosto de 2007, lo confirmó con declaración que los honorarios cuyo pago se ordena se reducen a la suma de $\$ 20.000 .000$. En contra de esta última sentencia, la parte vencida interpuso recurso de casación en el fondo aduciendo que la sentencia impugnada infringía la norma contenida en el artículo 665 del CPC. Arguye la recurrente que no fue rendida prueba alguna tendiente a acreditar el valor o cuantía de los servicios prestados, por lo que la estimación que efectuó el demandante en su líbelo, como también la ordenada pagar en el fallo, carece de toda fundamentación fáctica, sin que se acreditara previamente el valor o cuantía de los servicios prestados. Arguye igualmente que en el laudo y

Ya nos hemos ocupado en detalle del análisis de la mayor parte de las sentencias que se han pronunciado sobre esta materia, despejando varias de estas interrogantes, de manera que este comentario pretende complementar el estudio que ya se ha comenzado a delinear sobre esta materia. Sobre el particular, Ibid., pp. 188-195.

Número descriptor Microjuris: MJJ18454. 
la ordenata no se estableció honorario alguno, siendo esa la oportunidad precisa para hacerlo, por lo que se ha vulnerado -aduce- la normativa legal vigente. Finalmente la recurrente expresa que contra la sentencia arbitral que sirve de fundamento al presente juicio, existe pendiente una causa sobre nulidad de derecho público basada en que el árbitro demandante dictó sentencia fuera del plazo legal, es decir, después de los dos años de la aceptación del cargo, por lo que carecía de jurisdicción al respecto.

Para resolver este recurso, la Corte Suprema tuvo en cuenta- en lo medular- las siguientes circunstancias: 1. Que la demandada reconoció que el actor prestó servicios en calidad de árbitro, aunque -según aduce la demanda- no habría cumplido lo encomendado por los litigantes en cuanto a disolver y liquidar la sociedad; 2. Que el juez de primer grado tomó en cuenta diversos documentos acompañados a la causa en que el demandante actuó como juez árbitro; 3. Que era la sociedad demandada a la que correspondía acreditar el pago de los honorarios al actor o que los servicios que aquél prestó no lo fueron conforme a sus facultades o que no realizó el encargo, carga que la demandada no cumplió; 4. Que, asimismo, la Corte de Apelaciones de Chillán tuvo por acreditada que el demandante se desempeñó como juez árbitro en los autos iniciados en el año 2000, en los que se efectuaron 10 comparendos ordinarios, una absolución de posiciones, una designación de perito contable y una inspección personal del tribunal, dictándose la correspondiente sentencia en noviembre de 2002; 5. Si la remuneración de los servicios profesionales no se estableció por la convención de la partes, la ley o la costumbre, puede ser fijada por el juez, determinación en la que el tribunal debe considerar las diversas circunstancias que pueden influir en su regulación a fin que sea equitativa y corresponda a la importancia del servicio. 6. Se valoró especialmente que la sentencia dictada por el árbitro en su oportunidad tuvo el carácter de firme y ejecutoriada, lo que lleva a concluir que el juez árbitro cumplió con lo que le fue encomendado por los litigantes. 7. No se atribuyó importancia al hecho que en la actualidad se estuviera tramitando una nulidad de derecho público relativo al juicio arbitral, atendido lo inconcuso que resulta el que esa causa arbitral se inició, tramitó y falló por sentencia firme, lo que denota el cumplimiento del encargo por parte del árbitro. 8. Por otra parte, el sentenciador aclara que la norma del art. 665 del CPC, cuya contravención se invoca, establece que "en el laudo podrá hacer el partidor la fijación de su honorario, y cualquiera que esa su cuantía, habrá derecho para reclamar de ella. El precepto trascrito utiliza la vos "podrá" que sólo cabe entender en su exacto alcance, esto es, en cuanto le otorga a aquél la facultad potencia o posibilidad para determinar el monto de sus emolumentos conjuntamente con el fallo del asunto que le haya sido encargado, facilidad que ha de ser entendida en cuanto tal, y no a manera de una imposición sujeta a un instante preclusivo. En otras palabras, constituye una disposición que el legislador establece a favor del árbitro, considerando lo propicio que resulta el momento de dictar sentencia para que este juez informe los honorarios que le corresponden, cuya cuantía admite la reclamación que la misma norma prevé. De esta manera, el hecho que el árbitro no hubiera fijado sus honorarios en la sentencia, no es óbice para que no pueda fijarlos por cuerda separada. Por todo lo anterior, se desestima el recurso de casación en el fondo. 
Determinación de los honorarios del arbitro desde la óptica obligacional:

Problematización del estadio actual y propuesta de soluciones.

\section{Reflexiones y comentarios.}

\section{A. Reflexiones sobre el fallo en comento.}

Esta sentencia debe llamar nuestra atención a lo menos sobre tres puntos:

1. Las partes discuten sobre la exacta aplicación del Art. 665 del CPC y el sentenciador funda su fallo en tal precepto. En nuestra opinión, tal norma no debió aplicarse pues el ámbito de aplicación de esta norma sólo es extensiva a los juicios de partición ${ }^{8}$. En la especie se trataba de un juicio distinto, como es la liquidación y resolución de conflictos de una sociedad, por lo que la norma sólo pudo tener cabida por analogía.

2. De la mano de lo anterior, el sentenciador brinda una significancia muy distinta de esta norma, pues entiende y arguye que el árbitro debe "informar" en la respectiva sentencia los honorarios que le corresponden, cuestión que dista diametralmente de la realidad, pues lo que verdaderamente permite a los árbitros es "fijar sus honorarios" en la sentencia de un juicio particional. El mérito de este precepto consiste en presumir que las partes aceptan la determinación que el árbitro hace si no reclaman en contra de su monto dentro del plazo de quince días fatales desde que se notifica el laudo, de manera que su sólo silencio importa aceptación. Ergo, si las partes no están de acuerdo con la aludida estimación podrán oponerse en el aludido plazo, lo que obliga a resolver esta materia al tribunal de alzada que debe conocer de la apelación del laudo (si la hubiere) en única instancia.

Como se comprenderá, la diferencia no es puramente semántica, pues si se verbaliza esta acción sólo como una "información", las partes no tendrían oportunidad alguna de oponerse a la misma, sino que se estimaría desde luego como una sentencia firme e incuestionable, lo que ciertamente se aleja de la realidad, pues ello implicaría aceptar que árbitro puede ser juez y parte de sus propios honorarios ${ }^{9}$. Frente a ello se debe considerar, adicionalmente, que aun cuando el árbitro pueda fijar sus honorarios en el respectivo laudo, ello no implica de manera alguna que tal fijación pueda revestir el carácter de sentencia, en otras palabras, ello no muta su verdadera naturaleza, cual es la de un "convenio entre el partidor y los interesados"10.

$8 \quad$ Así también lo ha declarado la CA de Santiago, con 22 de enero de 1997, en causa "López Navarrete, Fernando con Consorcio General de Seguros S.A.",

9 Nuestros tribunales han aceptado esta doctrina en diversos fallos. A modo de ejemplo, Vid. CS. Revista, t. X, sec. $1^{\text {a }}$; CS, Revista, t. XXXIX, sec. $1^{\text {a }}$, p. 528; CA de Santiago, Revista, t. VIII, sec. 2a , p. 51; C. Valdivia, Gaceta, 1926, t. II, No 724; CA Concepción, Gaceta, 1937, t. II, No 173, p. 678.

10 De acuerdo a ello se ha fallado que la apelación deducida contra el laudo no importa reclamación contra el honorario del partidor que en él se fija, con lo que se evidencia que la determinación de la cuantía de su honorario que el árbitro hace en su sentencia no constituye resolución judicial, ni es parte integrante del laudo, así como la reclamación que las partes pudieren formular no es verdaderamente un recurso procesal. Vid. CA Santiago, Revista, t. VIII, secc. $2^{\circ}$, p. 51. 
3. Ahora bien, al determinar los honorarios del árbitro el sentenciador plantea que se deben considerar las diversas circunstancias que pueden influir en tal regulación a fin que sea equitativa y corresponda a la importancia del servicio; no obstante, al revisar el fallo, lo cierto es que aquél adolece precisamente de estos fundamentos. En efecto, para cuantificar los honorarios el sentenciador otorga un valor añadido a hechos de igual significancia y que corresponden tan sólo al presupuesto obligacional de las partes, como son: la resolución del litigio y el número de actos procesales que se realizaron en el juicio para llegar a este punto. En otras palabras, la cuantificación parece realizarse sobre la base de un único criterio, esto es, que el árbitro cumplió su obligación de comportarse como tal, lo que en nuestra opinión sólo pudo ser valorado como la causal obligacional que legitima la contraprestación de los honorarios, en tanto que si no se comportó como árbitro o no cumplió la obligación contraída, ningún honorario podría corresponderle. Frente a ello se debe obrar con precaución pues el árbitro podría no llegar a dictar sentencia e igualmente ser acreedor de honorarios, si por ejemplo, el término de sus funciones anticipado le es inimputable ${ }^{11}$.

De este modo, comprobado el presupuesto obligacional, la cuestión se centra en la determinación y cuantificación de los respectivos honorarios. ¿Cuáles son los criterios que habrán de considerarse? Tal estimación debe atender a criterios, pero éstos no deben obedecer sólo a aspectos formales, como el número de gestiones que lleva a cabo, sino que principalmente de contenido, es decir, "sobre cómo efectuó el encargo", pues la lógica nos indica que el quehacer mismo de la función que se el encomienda es actuar y comportarse como juez entre las partes hasta la completa decisión de los asuntos comprometidos o a la ejecución de tales resoluciones si fuere pertinente y a poner en el cumplimiento de esta tarea la diligencia que exige la ley. De esta manera, no se trata sólo de una obligación de resultados, sino principalmente de medios, por lo que se deben valorar las directrices tenidas en cuenta por el árbitro al cumplir su cometido en cuanto podría suceder que su prestación sea defectuosa aunque finalmente dicte el respectivo laudo.

En efecto, el deber de dictar el laudo corresponde a la obligación principal asumida por el árbitro, pero no se trata de emitir cualquier decisión jurídica de la contienda, sino que de una

11 Estos honorarios se deberán incluso si el árbitro no ha terminado su encargo, cuando los motivos no le sean imputables a éste, en este caso, se deberán en proporción al trabajo realizado. Si el árbitro, en cambio, nada realiza o le son imputables las causas de su mora, las partes podrían excusarse de cumplir esta obligación aduciendo la excepción de contrato no cumplido (Art. 1552 y 1489 CC). A este respecto, existe un fallo de la CA de Valparaíso, que data de fecha 6 de octubre de 2003, en causa "Mahan; con Sociedad de Inversiones e Inmobiliaria Cerro Castillo Ltda.". Asimismo, las partes no pueden excusarse de efectuar dicho pago, aduciendo que la sentencia por éste dictada no se encuentra ejecutoriada. Así lo ha entendido la CA Presidente Aguirre Cerda, que con fecha 9 de junio de 2000, en causa "Montimedina, Juan Pablo con Hilandería Las Américas S.A.”, y la CA de San Miguel, con fecha 19 de junio 2000. 
Determinación de los honorarios del arbitro desde la óptica obligacional:

Problematización del estadio actual y propuesta de soluciones.

decisión y solución específica que debe darse como resultado y culminación de un proceso arbitral que respetó unos principios concretos y siguió unos pasos y actuaciones determinadas, de manera que el resultado que se persigue no es tan sólo material. De este conjunto de deberes, unos son meramente instrumentales para llegar al fin del camino y al pronunciamiento del laudo, mientras otros son verdaderas obligaciones de fondo. Para que la obligación se entienda cumplida, deben operar otros criterios como la dictación oportuna o dentro de plazo, haber afinado el procedimiento a seguir por medio de reglas claras, notificadas a las partes, haber operado la imparcialidad e independencia, haber sido diligente en la aplicación de las normas jurídicas si ello correspondía, entre otros. Igualmente, el monto del litigio (cuantía económica) y la complejidad del asunto, podrían convertirse en otros punto a considerar, pues ningún sentido tendría que una sociedad pequeña que pretende resolver un litigio de una cuantía menor, termine pagando honorarios excesivos.

El sentenciador no repara en estos y otros hechos al cuantificar los honorarios del árbitro. No arguye otros fundamentos que los señalados precedentemente, lo que evidentemente provoca infinita seguridad en los litigantes al no estar claro el principal foco conflictual de este asunto, como es la determinación de los necesarios criterios a tener en cuenta en esta materia.

\section{B. Comentarios finales.}

Los honorarios nacen de la relación contractual habida entre el árbitro y las partes y corresponden a la principal obligación asumida por aquéllas. Existe un vacío sobre la forma y modo de fijarlos, pero de manera general se ha entendido que se puede realizar: 1 . Por acuerdo anticipado y voluntario de las partes y el árbitro, antes que éste último entre en sus funciones; 2. Por la determinación que formulen los árbitros en la sentencia de un juicio particional (art. 665 CPC), si las partes no se oponen a los mismos dentro del plazo respectivo; 3. Por la fijación que el árbitro efectúe en la sentencia fuera de los casos ya indicados, si las partes le han facultado para estos efectos y siempre que no reclamen de su fijación ${ }^{12}$; y 4. Por sentencia de los tribunales ordinarios al resolver el cobro de honorarios impetrado por el árbitro respectivo. En ningún caso el árbitro podrá fijar sus honorarios de manera unilateral ${ }^{13}$.

12 La jurisprudencia ha entendido que las partes podrían facultar al árbitro para que éste fije sus honorarios al final del juicio, pero en este caso el árbitro deberá esperar el cumplimiento total del encargo, es decir, deberá dictar sentencia en el arbitraje y notificar a las partes de ella, si así no lo hiciere, éste cometería falta sancionable por vía disciplinaria. Así lo ha expreso al CS, con fecha 4 de septiembre de 1995, en causa "Sociedad inmobiliaria las lilas S.A.".

13 Al respecto la CS ha señalado, con fecha 23 de junio de 1981, en causa "Soc. Enrique Zaror y Cía. Ltda.”, que no existe disposición alguna que autorice al árbitro arbitrador para fijar por si y ante sí sus honorarios en el juicio arbitral, ni tampoco aparece del expediente agregado que las partes lo facultaran al efecto; luego, al emitir el juez recurrido el indicado pronunciamiento, para el cual carecía de competencia, cometió una falta que debe corregirse por la vía disciplinaria. No obstante ello, el árbitro si podría proponer los honorarios en su sentencia y esperar a que las partes los acepten para que de esta manera que configure la obligación. La CS, con fecha 15 
Si bien los honorarios de los árbitros constituyen un asunto de índole enteramente privada, en tanto en principio afecta a los árbitros y a las partes que litigan ante él, lo cierto es que su carente marco legislativo provoca dos problemas que no son nada inocuos y que abandonan necesariamente la esfera de lo particular. El primero es que su falta de regulación convencional y/o legal implica necesariamente que aquéllos deban ser fijados por la justicia ordinaria con lo que los recursos involucrados (tiempo, recursos del tribunal, etc.) pasan a ser estatales; en segundo lugar, la dictación de sentencias poco satisfactorias en términos de fundamentos termina por mermar la seguridad jurídica y con ello la confianza que pudo haberse adquirido respecto de la institución arbitral. A estas motivaciones, debemos añadir la necesidad que el legislador se ocupe de la institución arbitral desde un punto de vista integral, donde los honorarios de los árbitros serán un punto reducido de la misma, aunque no por ello de menor importancia.

En definitiva nuestra propuesta en la materia pasa por establecer un marco normativo que permita delimitar cuáles son las obligaciones y derechos generadas para las partes y los árbitros, su ámbito de aplicación, requisitos, cuándo y cómo se dará cumplimiento a las mimas y qué tipo de responsabilidad se deriva de su incumplimiento. En relación a los honorarios, nos parece necesario recoger una práctica de Derecho comparado que se encuentra en proceso de expansión en cuanto, a falta de regulación convencional de los honorarios, se obligue a las partes a proveer, al inicio del juicio arbitral, fondos para pagar los honorarios de los árbitros. Para ello el árbitro y las partes debieran estimar los citados honorarios y gastos del procedimiento en atención a la cuantía económica del mismo y de las circunstancias que consideren relevantes y sobre dicha base se fije una provisión de fondos por la totalidad del importe estimado repartido por mitad. Ello no podrá obstar a que en el curso del arbitraje se puedan convenir nuevas provisiones en el caso que la cuantía de las pretensiones de las partes se incrementen o en función de nuevos factores que influyan en la dificultad, responsabilidad y disponibilidad que el procedimiento arbitral, así como a la fijación convencional de los honorarios que en definitiva tenga lugar (ciertamente esta provisión tendrá algún grado de simetría). Si ello no ocurriere, el texto legal debiera contar con criterios más o menos objetivos que impulsen una correcta determinación de los honorarios de los árbitros por parte de la justicia ordinaria que no atiendan a cuestiones puramente formales.

de diciembre de 1982, en causa “Angel Lampasona Vasallo”, así lo expresó al señalar que “... dicha decisión del árbitro sólo pudo plantearla en la sentencia como una proposición de honorarios". Tal resolución, sin embargo, no podría declarar que los honorarios se tendrán por aprobados con citación si las partes no se opusieren a ellos dentro del plazo respectivo, pues la misma constituiría una determinación de honorarios y no una proposición, tanto más cuando aquellos honorarios quedarían definitivamente determinados si no se formulase objeción. 
Determinación de los honorarios del arbitro desde la óptica obligacional:

Problematización del estadio actual y propuesta de soluciones.

\section{Anexo: Transcripción de la sentencia.}

Santiago, 1 de octubre de 2008.

VISTOS: En este juicio sumario sobre cobro de honorarios en juicio, Rol No 47.7092004, caratulado "Niño Morales, Dictino c/ Almonte Puentes, Daniel y otros" seguido ante el Primer Juzgado Civil de Chillán, la juez subrogante de dicho tribunal, Sra. Dilys Araya Couch, por sentencia de 25/5/2005, que se lee a fojas 56, acogió la demanda, ordenando a la demandada pagar al actor la suma de $\$ 50.000 .000$, reajustados conforme a la variación del Índice de Precios al Consumidor, entre la fecha de la sentencia y su pago efectivo, con costas.

En contra de dicho fallo, la parte demandada interpuso recurso de apelación y la Corte de Apelaciones de Chillán, por sentencia pronunciada el 3/8/2007, escrita a fojas 60, lo confirmó, con declaración que los honorarios cuyo pago se ordena se reducen a la suma de $\$ 20.000 .000$. En contra de esta última sentencia, la demandada ha interpuesto recurso de casación en el fondo.

Se trajeron los autos en relación.

CONSIDERANDO: PRIMERO: Que el recurrente sostiene en su libelo de nulidad sustancial, que la sentencia impugnada infringe la norma contenida en el artículo 665 del Código de Procedimiento Civil, disposición que establece que la determinación de los honorarios del Partidor, cualquiera sea su cuantía, debe efectuarse en el laudo.

Explica la recurrente, que no fue rendida prueba alguna tendiente a acreditar el valor o cuantía de los servicios prestados, por lo que la estimación que efectuó el demandante en su libelo, como también la ordenada pagar en el fallo, carece de toda fundamentación fáctica, sin que se acreditara previamente el valor o cuantía de los servicios prestados, habida consideración que en el laudo y ordenata no se estableció honorario alguno, siendo esa la oportunidad precisa para hacerlo, por lo que por ese capítulo, la sentencia de segundo grado ha vulnerado la normativa legal vigente. Tampoco es acertado, prosigue el recurso, que se estableciera que correspondía a la demandada acreditar el pago de los honorarios del actor, puesto que para ello resultaba indispensable que primero se estableciera la cuantía de éstos, lo que no se hizo. $\mathrm{Al}$ final de sus argumentos, la recurrente expresa que contra la sentencia arbitral que sirve de fundamento al presente juicio de cobro de honorarios, existe pendiente una causa sobre nulidad de derecho público, basada en que el árbitro demandante dictó sentencia fuera del plazo señalado en la ley, vale decir, después de dos años luego de la aceptación del cargo, por lo que carecía de jurisdicción al respecto; circunstancias por las que la sentencia que se dicte en esos autos sobre nulidad, resulta trascendental para dirimir correctamente la procedencia de los honorarios del árbitro.

Por último, expresa la recurrente, el error de derecho en que han incurrido los sentenciadores de segunda instancia, ha influido substancialmente en lo dispositivo de la sentencia, puesto que de haberse aplicado correctamente las disposiciones legales que reglamentan esta materia, a las cuales se ha hecho referencia precedentemente, habrían concluido que en la especie procedía rechazar la demanda (sic). 
SEGUNDO: Que para resolver este recurso es menester tener presente las siguientes circunstancias y antecedentes del proceso: a.- Que don Dictino Niño Morales, en conformidad con lo dispuesto en el artículo 697 del Código de Procedimiento Civil, presentó demanda de cobro de honorarios profesionales en juicio, en contra de la Sociedad Inmobiliaria Emmanuel S.A.C., en atención a su desempeño en calidad de Juez árbitro arbitrador, en el juicio seguido entre un centenar de accionistas y la referida sociedad; expresó que no obstante su prolongada labor profesional en dichos autos, no le han sido pagados sus honorarios profesionales por tal actuación, los que estima, de acuerdo a la cuantía de lo discutido y la labor realizada, en la cantidad de $\$ 50.000 .000$, más reajustes e intereses desde la fecha de la demanda, hasta su pago efectivo, con costas; b.- Que en su contestación, la demandada solicitó el rechazo de la demanda. Reconoció que el actor prestó servicios en calidad de árbitro, pero no cumplió - de acuerdo a lo encomendado por los litigantes - con la facultad de disolver y liquidar en su caso esta sociedad?; agregó que el demandante fijó sus honorarios en una suma desproporcionada en un juicio que, en términos generales, es de cuantía indeterminada, los que, tampoco, fijó en el respectivo laudo y ordenata, por lo que deben ser determinados por el tribunal que conoce de la demanda y luego de eso, establecer el derecho del actor para cobrarlos. Señaló, además, que, si bien la sentencia pronunciada en el arbitraje se encuentra firme, está siendo objeto de un juicio de nulidad de derecho público, en el que se cuestiona la existencia del tribunal arbitral; c.- Que el fallo de primer grado, teniendo en cuenta que de los documentos acompañados a la causa consta que el demandante actuó como Juez árbitro en el juicio seguido entre los accionistas y la sociedad demandada, concluyó que correspondía a ésta acreditar el pago de los honorarios al actor o, que los servicios que aquél prestó no lo fueron conforme a sus facultades, es decir, que no realizó el encargo como lo alegó, carga con la cual la demandada no cumplió, por lo que resolvió acoger la demanda, fijando los honorarios demandados en la suma pedida, sin intereses, por no tratarse de una convención en la que se haya estipulado su pago o de una operación de crédito de dinero que los genere y, en cuanto a los reajustes, se dispuso su cálculo a partir de la fecha de la sentencia, hasta su pago efectivo;

d.- Que contra ese fallo, la demandada dedujo recurso de apelación; e.- Que la Corte de Apelaciones de Chillán, conociendo del recurso señalado en el apartado que precede, con el mérito del expediente sobre disolución y liquidación de sociedad que trajo a la vista, tuvo por acreditado que el demandante se desempeñó en calidad de Juez árbitro en esos autos iniciados el 28/8/2000 y en los que se efectuaron diez comparendos ordinarios, una absolución de posiciones, una designación de perito contable y una inspección personal del tribunal, dictándose la correspondiente sentencia el 11/11/2002 y, teniendo presente que en caso que la remuneración de los servicios profesionales no se haya establecido por la convención de las partes, la ley o la costumbre, debe ser fijada por el juez, determinación en la que el tribunal debe considerar las diversas circunstancias que puedan influir en su regulación, a fin de que sea equitativa y corresponda a la importancia del servicio, atendiendo a los puntos de vista tanto del profesional, como del beneficiado, confirmó la sentencia apelada, con declaración que se reducen los honorarios demandados a la suma de \$20.000.000.

TERCERO: Que, asimismo, es pertinente dejar asentado que, entre los antecedentes a la 
Determinación de los honorarios del arbitro desde la óptica obligacional:

Problematización del estadio actual y propuesta de soluciones.

vista, se cuentan las compulsas de la causa Rol No 43.263-S, caratulada "Sociedad Inmobiliaria Emmanuel S.A. con Umaña Lezana Manuel y otros", seguida sobre disolución y liquidación de Sociedad Inmobiliaria Emmanuel S.A., ante el Juez árbitro don Dictino Niño Morales, en la cual se pronunció sentencia definitiva con fecha 11/11/2002, fallo arbitral que se abocó al objeto del juicio determinado en el primer comparendo de la causa celebrado el 30/8/2000? la resolución de los conflictos sociales, disolución y liquidación, en su caso, de la aludida sociedad inmobiliaria - en contra del cual los demandados recurrieron de apelación, arbitrio procesal que, aunque concedido, no llegó a ser conocido propiamente por el Tribunal de Alzada de Chillán, toda vez que a su respecto se dedujo un recurso de hecho al que la misma Corte hizo lugar por sentencia de 14/3/2003, y declaró inadmisible el antedicho recurso de apelación interpuesto en contra de la sentencia definitiva dictada en los referidos autos arbitrales. Con lo anterior, entonces, aquella sentencia por la que el Sr. Juez árbitro resolvió el asunto que fue objeto del juicio para el que fue designado, adquirió el carácter de firme o ejecutoriada, lo que lleva a concluir que el juez árbitro designado cumplió con lo que le fue encomendado por los litigantes, adquiriendo pleno vigor la pretensión de obtener que sus servicios sean remunerados por las partes en el proceso que llevara adelante; sin que presente mayor relevancia el estado procesal de una causa diversa sobre nulidad de derecho público relativa al juicio arbitral al que conciernen los honorarios cuyo cobro se persigue en autos, atendido lo inconcuso que resulta el que esa causa arbitral se inició, tramitó y falló por sentencia firme que denota el cumplimiento del cometido encargado al tribunal;

CUARTO: Que, ahora bien, atendiendo a los errores de derecho que la recurrente denuncia como fundamento de la nulidad sustancial que postula, resulta conveniente recordar que la norma contenida en el artículo 665 del Código de Procedimiento Civil, cuya contravención invoca quien recurre, establece que "En el laudo podrá hacer el partidor la fijación de su honorario, y cualquiera que sea su cuantía, habrá derecho para reclamar de ella. La reclamación se interpondrá en la misma forma y en el mismo plazo que la apelación, y será resuelta por el tribunal de alzada en única instancia".

QUINTO: Que, como se observa, el precepto trascrito en el motivo anterior, al referirse a la fijación de honorarios del árbitro en su laudo, utiliza la voz "podrá", forma verbal que sólo cabe entender en su exacto alcance, esto es, en cuanto le otorga a aquél la facultad ? potencia o posibilidad? para determinar el monto de sus emolumentos conjuntamente con el fallo del asunto que le haya sido encargado; facilidad que ha de ser entendida en cuanto tal, y no a manera de una imposición sujeta a un instante preclusivo. En otras palabras, constituye una disposición que el legislador establece a favor del árbitro, considerando lo propicio que resulta el momento de dictar sentencia para que este juez informe los honorarios que le corresponden, cuya cuantía admite la reclamación que la misma norma prevé.

SEXTO: Que, del texto de la sentencia arbitral pronunciada el 11/11/2002 en causa Rol No 43.263-S, caratulada "Sociedad Inmobiliaria Emmanuel S.A. c/ Umaña Lezana, Manuel y otros", se observa que el actor no hizo en ella la determinación de sus honorarios; omisión que no es óbice para llegar a fijarlos por cuerda separada.

SÉPTIMO: Que, para el caso que nos ocupa, tras haber cumplido con lo encargado en los 
autos arbitrales, empero sin haber fijado la cuantía de su remuneración al dictar el laudo, el actor inició el presente juicio para obtener el cobro de sus honorarios, cuya procedencia han admitido los jueces, tanto de la primera, como de la segunda instancia, aunque en cuantías diversas, decisión que no importa la infracción normativa que la demandada pretende en su recurso de nulidad, atendido que, como ya se ha expresado, lo facultativo de la redacción del precepto contenido en el artículo 665 del Código de Procedimiento del ramo, permite que, para el caso que el juez árbitro interesado no ejerza la posibilidad concedida por la ley, pueda de igual modo obtener la fijación de su remuneración por la vía procesal pertinente, mediante el ejercicio de su derecho de petición, dentro de los plazos generales y régimen de recursos previstos en la legislación atinente.

OCTAVO: Que, en todo caso, la decisión adoptada se efectúa según los hechos y situación jurídica actualmente vigente, sin que se deba tener presente resultados eventuales de impugnaciones en actual tramitación.

NOVENO: Que en estas condiciones, no habiéndose incurrido en el error de derecho denunciado, debe desestimarse el recurso de casación en el fondo. Por estas consideraciones y visto, además, lo dispuesto en los artículos 764, 765, 766, 767 y 768 del Código de Procedimiento Civil, se rechaza el recurso de casación en el fondo deducido en lo principal de fojas 84, por don Juan Antonio de la Hoz Fonseca, en representación de los demandados, contra la sentencia de 3/8/2007, escrita a fojas 82 vuelta.

Regístrese y devuélvase, con sus agregados.

Redacción a cargo de la Ministro Sra. Margarita Herreros Martínez. $\mathrm{N}^{\circ} 4739-07$.

Pronunciado por la Primera Sala de la Corte Suprema, Integrada por los Ministros Sres. Milton Juica A., Sergio Muñoz G., Sra. Margarita Herreros M. y Sr. Hugo Dolmestch U. y Abogado Integrante Sr. Hernán Álvarez G. 